first meeting, and Douglas Anderson is the moderator of the second meeting, consisting of 7 cases of angle closure glaucoma. The panels consist of eminent researchers into glaucoma and are different in each symposium (except for Robert Shaffer, who was present in both, and whose comments are always to the point, profound yet essentially practical). The case presentations are halted at certain points for discussions and generalisations, and much ground is covered. The bias of the symposium is towards the solution of specific everyday problems, and, not unexpectedly, the level of discussion varies widely between traditional views and controversial and stimulating statements.

It is clearly impossible to discuss the 19 chapters individually, but perhaps a few statements and opinions may be singled out for their interest. Assessment of the diurnal variations in pressure is best carried out by sporadic checks at the clinic at different hours, or by home tonometry; inpatient hospital phasing is not so reliable, as it involves a profound change in the patient's routine. Almost all the panelists commonly used the water drinking test, but tonography is no longer a routine investigation, being reserved for special cases. A recent spate of litigation cases in the US concerning juvenile glaucoma prompts several panelists to perform tonometry on every co-operative subject regardless of age. However, Shaffer would limit this to cases with suspicious-looking discs and to those persons in whom it can be carried out in a reasonably short time.

Epinephrine almost certainly improves outflow facility fairly rapidly and not, as was thought, after a long latent period. The peak of its dose-response curve appears to be at the $1 \%$ concentration of active drug in the short term, though it may be as high as $2 \%$ in the long term (Bernard Becker). Stability of adrenaline solution is greater with the bitartrate and the hydrochloride (Epifrin, Glaucon) than with the borate (Epinal, Eppy), but the borate compounds are more comfortable to use. Although little statistical evidence has been adduced, there is an impression that the disc cup changes more readily if it is already large (John Hetherington). In ocular hypertension the pressure responses to, and patient tolerance of, treatment are important factors in deciding when and how much to treat. In drainage operations vitreous aspiration is increasingly recommended to prevent sealing up of the drainage aperture by vitreous. Provocative tests in angle closure glaucoma remain essentially unreliable. The least unsatisfactory tests are the dark room test and the prone test. Mydriatic tests give many false positives. Neither the patient's advanced age nor a considerable degree of widening of the angle with pilocarpine should weaken our resolve to operate on every case of acute angle closure glaucoma (Pollack). However, tonography has a place in evaluating the chances of success of peripheral iridectomy after an acute attack: if $\mathrm{C} \leqslant 0 \cdot 1$, we can expect over $75 \%$ of failures (Shaffer).

The volume is beautifully presented and contains several illustrations and clear tables.

JOHN ROMANO

Mechanisms of Colour Vision. By W. S. Stiles. 1978. Pp. 287, figs., tables, refs. Academic Press, London (£14)

This book contains some of the important papers published during the last half century by Dr W. S. Stiles, a doyen amongst visual physicists. The title is slightly misleading, because the author's wide erudition has enabled him to range not only over the mechanisms of colour vision but also over those of vision in general. The papers are reproduced photographically, and in an introductory chapter there is an indication of the extent to which the author may have wished to modify earlier views. Some of the papers may be hard to trace in the original, and for this reason newcomers to the field have every reason to be grateful for the appearance of the book. Its method of production, however, entails repetition, and this may cause a justifiable grumble on economic grounds.

\title{
Correspondence
}

\section{On difficulties in deciding the aetiology of cataract}

TO THE EDITOR, British Journal of Ophthalmology

SIR, Because the lens fibre system contains only 1 type of cell it has a fairly limited number of ways in which it can respond to injury and on interference with its nutrition. It is therefore extremely difficult from the appearance of the cataract, and the clinical examination of a few cases, to decide whether a history of exposure to a known cataractogenic agent is proof that this agent is important in the genesis of cataract in man, as has been implied, for instance, in the recent study by Zaret and Snyder (1977).

As an example of such a difficulty we may consider a patient employed in servicing radar equipment who had a history of intermittent exposure over a period of 20 years to $3-\mathrm{cm}$ microwaves from high-intensity sources of up to $60 \mathrm{~kW}$. He developed cataract at the age of 52 with reduced vision in each eye. Slit-lamp examination showed interesting features in both lenses. There was vacuolation of the anterior capsule and striate changes in the anterior cortex, while at the posterior pole there were vacuoles and a feathered appearance of lens fibres. No other changes in the eye were apparent, and the intraocular tensions were normal.

It was possible at a later date to examine these lenses by light and electron microscopy. There were large areas of fibre disruption at the anterior pole, and portions of the anterior epithelium appeared to have suffered some necrosis. In addition the superficial cataractous lens fibres were separated from the capsule by clefts filled with albuminous fluid. At the posterior pole the lens fibre membranes had produced myeloid whirls and numerous granular bodies. From these appearances, which can all be seen in senile cataract, it was not possible to state that the changes were character- 